\title{
Laparoscopic Ventral Mesh Rectopexy (LVMR) - an Early Experience in Birdem Hospital
}

\author{
Md. Ezharul Haque Ratan ${ }^{1}$, Hasina Alam²
}

\begin{abstract}
:
Background : Full thickness rectal prolapse are treated by multiple procedures through perineal and abdominal approach. Consensus is lacking as to the best option. Each procedure is associated with significant recurrence rate.

Objective : The aim of this study is to report the effectiveness, complications following laparoscopic ventral mesh rectopexy in patients with full thickness rectal prolapse.

Methods : The study is a retrospective evaluation of 6 consecutive patients by a single attending surgeon in a general and laparoscopic surgery unit,between July 2014 to June 2016. Peritoneum was incised at the pouch of Douglas or rectovesical pouch, space created between the rectum and the vagina or urinary bladder, polypropylene mesh was fixed to the rectum with non-absorbable suture and to the promontory of the sacrum with same suture instead of staples. In females, the mesh was also fixed anteriorly with posterior fornix of vagina. The peritoneum was suture closed over the mesh. Patients were reviewed at 1 and 6 months, then annually to assess recurrence, morbidity and mortality.
\end{abstract}

Result : There was no recurrence or mortality among four female and two male patients. Morbidity consistedof chronic deep perineal pain in one youngmale patient who was treated conservatively with oral analgesic.

Conclusion : LVMR seems to emerge as a safe and effective procedure to treat full thickness rectal prolapse, but large series and long term results are needed and we are continuing the study for the same.

Key words : Rectal prolapse, intraperitonealventral mesh rectopexy (IVMR), polypropylene mesh,pouch of Douglas.

\section{Introduction}

Full thickness rectal prolapse (FTRP) is an extrusion of full thickness of the wall of rectum beyond the anal verge ${ }^{1}$. Full thickness rectal prolapse are treated traditionally by procedures through perineal or abdominal approach. The perineal approach (Delorme and Altemeier Procedures) are becoming less favourite due to high recurrence rates. It is nowadays generally accepted that the abdominal procedures including the rectopexy to the promontory has a lower recurrence rate and improved functional outcome and are therefore preferred over the perineal operations ${ }^{2}$. Since 1995, the laparoscopic abdominal approach is practiced ${ }^{3,4}$ Laparoscopic rectopexy has less abdominal discomfort, faster recovery, shorter hospital stay, and absence of $\operatorname{scar}^{5,6}$.

1. Dr. Md. Ezharul Haque Ratan, MS (General Surgery), Associate professor, Surgery, Ibrahim Medical college and BIRDEM, General Hospital ,Shahbagh, Dhaka-1000, Bangladesh

2. Dr. Hasina Alam, FCPS (General Surgery), Registrar Surgery, Ibrahim Medical college and BIRDEM, General Hospital ,Shahbagh, Dhaka-1000, Bangladesh

\section{Corresponding Author:}

Dr. Md. Ezharul Haque Ratan

MS (General Surgery)

Associate professor, Surgery,

Ibrahim Medical college and BIRDEM

Room no-445, BIRDEM General Hospital

Shahbagh, Dhaka-1000, Bangladesh

Tel : +88 01711172547

Email: ezhar65@gmail.com
However, consensus is lacking as to the best option. Each procedure is associated with significant recurrence rate. The aim of this study is to report the effectiveness, procedural minutes and complications following laparoscopic ventralrectopexy (LVR) in patients with full thickness rectal prolapse.

\section{Materials and methods}

The study is a retrospective evaluation of 6 consecutive patients by a single attending surgeon in a general and laparoscopic surgery unitof BIRDEM general hospital, between July 2014 to June 2016. Four patients (67\%) were female and two patients (33\%) weremale. Mean age was $53.5 y r s$ (18-80yrs). All patients complained of something coming out of anus on straining, for several years (6yrs to 17yrs). All patients were able to reposition the prolapsed rectum manually. They also complained of mucus discharge and occasional pain. FTRP was diagnosed clinically in prone and squatting posture. Pre-operative workup included routine investigations for general anaestheia fitness. All patients were kept on low residual diet for 3 days pre-operatively and a laxative given day before surgery. A per-urethral Foley catheter was placed in situ just before surgery and removed at the end of procedure.Surgery was performed under general anesthesia. Laparoscopy was performed through 3 / 4 trocars, one $10 \mathrm{~mm}$ trocar in umbilicus, two $5 \mathrm{~mm}$ trocars in right and left iliac fossa. A fourth $5 \mathrm{~mm}$ trocar was inserted in suprapubic area where needed. Patient was in Trendelenburg position. Peritoneum was incised at the pouch of Douglas or rectovesical pouch, space created between the rectum and the vagina or urinary bladder, polypropylene mesh was fixed to the rectum with non-absorbable suture and to the promontory 
of the sacrum with same suture instead of staples.In females, the mesh was also fixed anteriorly with posterior fornix of vagina. .The peritoneum was suture closed over the mesh. Rest of the surgery was completed in usual manner of laparoscopic cholecystectomy. Oral diet was resumed 24 hours after surgery. Data were collected on patients' age, sex, preoperative diagnosis, operative methods and length of stay in hospital. Patients were reviewed at 1 and 6 months, then annually to assess recurrence, morbidity and mortality.

\section{Results}

Six consecutive patients of FTRP underwent LVMR, in BIRDEM general hospital, between July 2014 to June 2016. Four patients (67\%) were female and two patients (33\%) were male. Mean age was 53.5yrs (18-80) years. All patients were diagnosed as FTRP clinically. Pre-operative workup included routine investigations. All patients underwent LVMR with polypropylene mesh. There were no intraoperative complications in any of the patients. All six patients had uneventful post-operative course.Oral diet was resumed 24 hours after surgery. The hospital stay of the patients ranged from 48 hours to 5 days (mean 3 days). Patients were reviewed at 1 and 6 months, then annually to assess recurrence, morbidity and mortality.There was no recurrence or mortality among the patients. Morbidity consisted of chronic deep perineal pain in one young male patient who was treated conservatively with oral analgesic.

\section{Discussion}

LVMR is the ideal treatment for internal and external rectal prolapse $^{7,8}$ with minimum complications and recurrences in comparison to open abdominal posterior rectopexy9. Our series consists of six patients which is very small in number compared to other studies ${ }^{4,5,7}$. However, similar to other studies, low complication and no recurrence was found. Improved results are possibly due to limited anterior rectal mobilization and no lateral mobilization. Thus preventing rectal denervation and post-operative constipation ${ }^{10}$. Proximal mesh fixation to sacral promontory prevents the rectal intussusception. In our series, majority were female (67\%), which resembles other series ${ }^{11}$. In fact, a low anterior dissection to levator ani corrects rectocele. This procedure allows correction of the median compartment with vaginal vault fixation to the mesh and is therefore the first choice of surgical treatment in females ${ }^{10}$. Low fixation of mesh to ventral rectal wall also minimize recurrence rates and allow repair of large rectoceles ${ }^{8}$. The creation of a shallow, elevated pouch of Douglas at the end of operation by suturing the peritoneal incisions over the mesh corrects a concomitant enterocele and sigmoidocele ${ }^{12,13}$. Age range in this series is 18-80 years which corresponds to other data in different series ${ }^{11}$. In our series, we have used synthetic mesh in all cases because it is less expensive and more readily available in our centre. However, different publications show similar rates of mesh complications and failure for biologic and synthetic meshes ${ }^{14}$. The hospital stay of the patients ranged from 48hours to 5 days (mean 3 days) as in different studies ${ }^{12}$. Patients were reviewed at 1 and 6 months, then annually to assess recurrence, morbidity and mortality. This follow-up period is quiet inadequate for such procedure. However, there was no recurrence or mortality among the patients. Morbidity consisted of chronic deep perineal pain in one young male patient who was treated conservatively with oral analgesic. More results will show in subsequent follow-up years.All large series ${ }^{5,711,12}$ have a minimum of 5 years of follow-up, which show that complications arise usually after 3 years.

The strength of the study is inclusion of all uncomplicated cases who were willing to undergo LVMR. Limitation of the study is, it is a retrospective study, number of cases is small and follow-up period is short.

\section{Conclusion}

LVMR seems to emerge as a safe and effective procedure to treat full thickness rectal prolapse, but large series and long term results are needed and we are continuing the study for the same.

\section{Table 1: Study variables}

\begin{tabular}{ll}
\hline Variables & Data \\
\hline Total number of patients & 6 \\
Age in years (Mean) & 53.5 years (18-80 years) \\
Male & $2(33 \%)$ \\
Female & $4(67 \%)$ \\
Duration of Hospital stay & 48 hours - 5 days (3 days) \\
Duration of Follow-up & 1 momths, 6 months, yearly
\end{tabular}

Table 2: Complications

\begin{tabular}{ll}
\hline Variables & Data \\
\hline Mortality & $0(0 \%)$ \\
Intraoperative Complication & $0(0 \%)$ \\
Postoperative Complication & \\
Deep Perianal Pain & $1(17 \%)$ \\
Recurrence & $0(0 \%)$ \\
\hline
\end{tabular}

\section{Reference}

1. Jones OM, Cunningham C, Lindsey I. The assessment and management of rectal prolapse, rectal intussusception, rectocoele, and enterocoele in adults. BMJ 2011; 342:325-9.

2. Schiedeck TH, Schwandner O, Scheele J, Farke S, Bruch HP. Rectal prolapse:which surgical option is appropriate? Langenbecks Arch Surg 2005;390:8-14

3. Auguste T, DubreuilA, Bost R, Bonaz B, Faucheron JL.Technical and functional results after laparoscopic rectopexy to the promontory for completr rectal prolapse. Prospective study in 54 consecutive patients. Gastroenterol Clin Biol. 2006;30:659-63.

4. Faucheron JL, Voirin D, Reche F, Dudreuil A. Technical results of the laparoscopic rectopexy to the promontory for total rectal prolapse: prospective evaluation in 100 consecutive patients. J Coeliochir. 2007;63:9-12. 
5. Solomon MJ, Young CJ, Eyers AA, Roberts RA. Randomized clinical trial of laparoscopic versus open abdominal rectopexy for rectal prolapse. Br J Surg. 2002;89:35-9.

6. Sajid MS, Siddqui MR, Baig MK. Open versus laparoscopic repair of full thickness rectal prolapse : a re-meta-analysis. Colorectal Dis. 2010;12:515-25

7. D'hoore A, Penninckx F.Laparoscopic ventral recto(colpo)pexy for rectal prolapse: surgical technique and outcome for 109 patients. SurgEndosc 2006; 20:1919-23.

8. Wijffels N, Cunningham C, Dixon A, Greenslade G, Lindsey I. Laparoscopic ventral rectopexy for external rectal prolapse is safe and effective in the elderly. Does this make perineal procedures obsolete? Colorectal Dis 2011; 13:561-6.

9. Sileri P, Franceschilli L, De Luca E. Laparoscopic ventral rectopexy for internal rectal prolapse using biological mesh: postoperative and short-term functional results. J Gastro-intestSurg 2012; 16:622-8.
10. Sileri P, ,Franceschilli L, De Luca E.Modified Laparoscopic Ventral mesh rectopexy. Tech Coloproctol 2014; 18:591-4.

11. Charles E, Pierpaolo S.A multicenter collaboration to assess the safety of laparoscopic ventral rectopexy. Dis colon rectum 2015;58:799-807

12. Boons P, Collinson R, Cunningham C, Lindsey I.laparoscopic ventral rectopexy for external rectal prolapse improves constipation and avoids de novo constipation. Colorectal Dis 2010;12:526-32.

13. Mercer-Jones MA, D'Hoore A, Dixon AR.consensus on ventral rectopexy: report of a panel of experts. Colorectal Dis 2014;16:82-8.

14. Van den EsschertJw, van Geloven AA, Vermulst N, Groenedijk AG, de Wit LT, GerhardsMF. Laparoscopic ventral rectopexy for obstructed defecation syndrome. SurgEndosc 2008; 22(12):2728-32. 